\title{
Periprosthetic joint infections: a clinical practice algorithm
}

\author{
LUIGI VOLPE ${ }^{1}$, PIER FRANCESCO INDELLI ${ }^{2}$, LEONARDO LATELLA ${ }^{1,3}$, PAOLO POLI $^{1}$, \\ JALE YAKUPOGLU ${ }^{4}$, MASSIMILIANO MARCUCCI ${ }^{1,3}$
}

1 Articular Replacements Excellence Center (CESAT) - Fondazione Onlus “...In Cammino...”, Fucecchio, Italy

2 New Mexico Veterans Affairs Health Care System (NMVAHCS) and Adult Reconstruction University of New Mexico, Department of Orthopaedics and Rehabilitation, Albuquerque, New Mexico, USA

3 Orthopedic Clinic, University of Florence School of Medicine, Italy

4 The Breyer Center for Overseas Studies, Stanford University in Florence, Italy

\begin{abstract}
Purpose: periprosthetic joint infection (PJI) accounts for $25 \%$ of failed total knee arthroplasties (TKAs) and $15 \%$ of failed total hip arthroplasties (THAs). The purpose of the present study was to design a multidisciplinary diagnostic algorithm to detect a PJI as cause of a painful TKA or THA.

Methods: from April 2010 to October 2012, 111 patients with suspected PJI were evaluated. The study group comprised 75 females and 36 males with an average age of 71 years (range, 48 to 94 years). Eightyfour patients had a painful THA, while 27 reported a painful TKA. The stepwise diagnostic algorithm, applied in all the patients, included: measurement of serum C-reactive protein (CRP) and erythrocyte sedimentation rate (ESR) levels; imaging studies, including standard radiological examination, standard technetium-99m-methylene diphosphonate (MDP) bone scan (if positive, confirmation by LeukoScan was obtained); and joint aspiration with analysis of synovial fluid.

Results: following application of the stepwise diagnostic algorithm, 24 out of our 111 screened patients were classified as having a suspected PJI (21.7\%). CRP and ESR levels were negative in 84 and positive in 17 cases; $93.7 \%$ of the patients had a positive technetium-labeled bone scan, and $23 \%$ a positive Leuko-
\end{abstract}

\section{Corresponding Author:}

Pier Francesco Indelli, MD, PhD

Adult Reconstruction UNM, Department of Orthopaedics and Rehabilitation, New Mexico Veterans Affairs Health Care System (NMVAHCS)

1501 San Pedro Drive SE, Albuquerque, NM 87108, USA

E-mail: pierfrancesco.indelli@unifi.it
Scan. Preoperative synovial fluid analysis was positive in $13.5 \%$; analysis of synovial fluid obtained by preoperative aspiration showed a leucocyte count of > 3000 cells $\mu / 1$ in $52 \%$ of the patients.

Conclusions: the present study showed that the diagnosis of PJI requires the application of a multimodal diagnostic protocol in order to avoid complications related to surgical revision of a misdiagnosed "silent" PJI.

Level of evidence: Level IV, therapeutic case series.

Key Words: algorithm, arthroplasty, diagnosis, infection, joint, TKA, THA.

\section{Introduction}

Total joint arthroplasty (TJA) is a highly successful procedure in the treatment of degenerative joint diseases. The number of total knee arthroplasties (TKAs) and total hip arthroplasties (THAs) is increasing dramatically due to the increase in the average life span of the population. It has been estimated that around 800,000 hip and knee TJAs are performed annually in the USA (1). Periprosthetic joint infection (PJI) accounts for $25 \%$ of failed TKAs and $15 \%$ of failed THAs $(2,3)$. The risk of septic loosening is estimated to be between 0.39 and $2.6 \%$ after primary TJA and between 8 and 12\% after revision TJA (3-5). In the case of a painful TJA it is mandatory to distinguish between aseptic and septic causes of the failure, given that the treatment for a PJI might-impact on the patient's general health status, necessitating prolonged hospitalization and repeated surgical interventions; a PJI can sometimes even result in definitive loss of the implant with significant permanent deformity. The 
final outcome after PJI treatment differs considerably from the outcome of primary TJA, as shown in the literature (6).

Predisposing factors to PJI include advanced age, malnutrition, obesity, diabetes, rheumatoid or psoriatic arthritis, simultaneous infectious foci in different anatomical districts, previous joint surgeries or an infection in a previous arthroplasty (1). Many predisposing factors are related to the index TJA procedure and include prolonged duration of surgery, delayed wound healing, persistent hematoma, and postoperative subcutaneous cellulitis. It is indeed well known that joint implant infection can arise through three different routes: direct infection at the time of the index procedure, hematogenic infection, and reactivation of latent infection (7).

Surgeons currently use a wide range of infection prevention strategies, including shorter and specific courses of antibiotic therapy, shorter duration of surgery, the use of less invasive surgical techniques, and, especially, better patient selection (8-10).

The clinical appearance of PJI depends on the virulence of the pathogenic agent, the nature of the infected tissue, the infection route, and the length of disease evolution. PJI might present acutely with severe pain, high fever, local warmness, and sometimes surgical wound secretions, while the presenting signs of chronic infections are progressive pain, the formation of cutaneous fistulae and/or drainage of purulent secretions. Currently, surgeons and internal medicine physicians seeking to diagnose PJI use a multidisciplinary test battery that includes: tests to detect local inflammation, such as synovial fluid white blood cell (WBC) count and synovial tissue histology (11); measurement of the levels of systemic markers of inflammation, such as serum C-reactive protein (CRP), erythrocyte sedimentation rate (ESR) and interleukin6 (IL-6) (12); imaging studies such as standard radiographs, technetium-labeled bone scans, magnetic resonance imaging, computed tomography (CT) and positron-emission tomography (13); and several bacterial isolation techniques (Gram stain, culture).

Because of the above-mentioned issues surrounding the early detection and treatment of PJI, several scientific societies in the field of orthopedics recently published algorithms on how to approach a painful TJA in the presence of a suspected PJI $(14,15)$.
The purpose of the present study was to present a multidisciplinary diagnostic algorithm designed to detect PJI as the cause of a painful TKA or THA. Use of this algorithm is recommended in particular in medical institutions not having the necessary resources to implement all the recommendations contained in the relevant international guidelines (16).

\section{Methods}

All the patients evaluated at our institution from April 2010 to October 2012 for possible infection of a painful THA or TKA were included in the present study. Patients affected by any form of systemic inflammatory disease and patients with a suspected adverse tissue reaction to metal debris were also included. Patients receiving antibiotics within three weeks of joint aspiration were excluded.

One hundred eleven patients met the inclusion criteria. The study group comprised 75 females and 36 males with an average age of 71 years (range 48 to 94 years). The males were younger (average age 64 years) than the females (average age 74 years). Eight-four patients had a painful THA, while 27 reported a painful TKA. Patient screening included a thorough history and physical examination. Information obtained from a patient's history included the type of prosthesis, date of implantation, past surgeries on the joint, history of wound healing problems, PJI risk factors, remote infections, current clinical symptoms (including the presence of pain during passive and active range of motion), drug allergies and intolerances, co-morbidities, prior microbiology results from previous arthrocentesis and surgical procedures, and previous antimicrobial therapies. The skin in the affected area was always screened for heat, rubor, cellulitis and possible surgical wound secretions.

The stepwise diagnostic algorithm for all patients included: systemic measures of inflammation, namely: serum CRP levels (positive if $>10 \mathrm{mg} / \mathrm{L}$ ) and ESR levels (positive if $>30 \mathrm{~mm} / \mathrm{hr}$ ); imaging studies, namely: standard radiological examination for THA (standing anterior-posterior view of the pelvis, standing anterior-posterior view and lateral view of the affected hip) or TKA [standing anterior-posterior view of the of the affected limb, standing anterior-posterior 
view and lateral view of the affected knee, and bilateral Merchant's knee view (17)], and technetium- ${ }^{99} \mathrm{~m}$ methylene diphosphonate $\left({ }^{99} \mathrm{~m}\right.$-Tc MDP) bone scan; if this test resulted positive for implant loosening, patients received a bone scan with ${ }^{99} \mathrm{~m}$-Tc antigranulocyte antibody Fab' fragments (LeukoScan) (18). Joint aspiration was performed in all the patients (fluoroscopically assisted in the THA cases) who suspended antibiotic therapy three weeks beforehand: analysis of synovial fluid included total cell count and differential leucocyte count and cultures for aerobic and anaerobic organisms, considering that two or more positive cultures with the same organism or a single positive culture with a virulent microorganism has acceptable sensitivity and specificity $(9,19)$.

The patients were considered on a single-case basis and the following features constituted definitive evidence of PJI: combined abnormal ESR and CRP levels, a positive ${ }^{99} \mathrm{~m}$-Tc MDP bone scan, a positive LeukoScan, and a positive synovial fluid analysis with a differential of $>65 \%$ neutrophils or a leucocyte count of $>3000 \mu / 1$, and the presence of two positive cultures with the same organism or a single positive culture with a virulent pathogen.

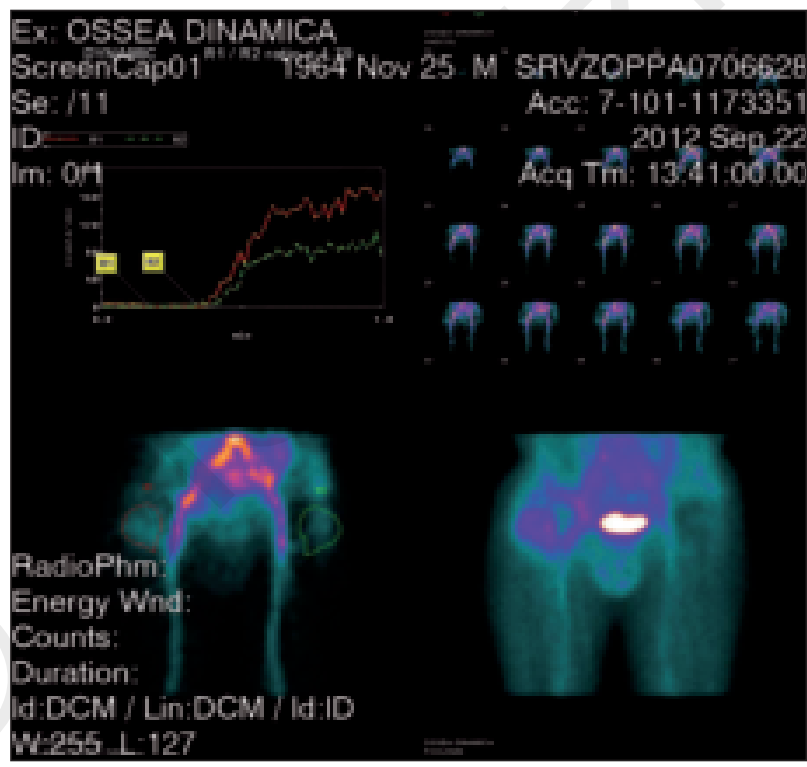

Fig. 1. A standard ${ }^{99} \mathrm{~m}$-technetium MDP bone scan showing hypercaptation in the right hip with aspecific inflammatory response. The analysis of the vascular phase shows hyper-caption of the contrast medium at the level of the trochanteric region and along the proximal femoral stem (SPECT CT technique).

\section{Results}

Following application of our stepwise diagnostic algorithm, 24 out of our 111 screened patients (8 men and 16 women with a mean age of 72 years) were classified as having a suspected PJI (21\%). This group comprised 10 THA (9\%) and 14 TKA (12.6\%) cases.

Serum CRP and ESR levels were negative in 84 cases and positive in 17 cases. A single positivity was identified in 10 cases (CRP in 7 and VES in 3): the average ESR value was $55 \mathrm{~mm} / \mathrm{hr}$ while the average CRP value was $47 \mathrm{mg} \backslash \mathrm{L}$.

All 111 patients underwent a technetium-labeled bone scan (Fig. 1), which was positive in 104 (93.7\%) and negative in seven (6.3\%). A leucocyte scan (LeukoScan) protocol (16) was applied to these 104 positive bone scans (Fig. 2) and 24 (23\%) resulted positive.

Synovial fluid obtained by preoperative aspiration in all 111 patients showed an average leucocyte count of 27,800 cells $\mu / \mathrm{l}$ (range: 1,084 to 77,193 ): a leucocyte count of $>3000$ cells $\mu / 1$ was measured in 58 cases (52\%). An average differential of $79.1 \%$ polymorphonuclear neutrophils (range: $50 \%$ to $99 \%$ ) was shown in this case series: 47 cases (42\%) (showed a differential of $>65 \%$ neutrophils. Fifteen cases $(13.5 \%)$ were found to be culture positive (13.5\%). The organisms isolated were: Staphylococcus (9 cases: Epidermidis in 4, Hominis in 2, Coagulase neg. in 2, Capitis in 1), Pseudomonas (1 case), Streptococcus Agalactiae (1 case), Corinebacterium ( 2 cases: Urealyticum in 1, Minutissimum in 1), FusobacteriumVarium (1 case), and a Bacillus species (1 case). Nine other patients $(8.1 \%)$ had negative cultures but because these patients showed high positivity on all other algorithm tests, the authors considered this subgroup affected by PJI. Analysis of synovial fluid from a sample taken intra-operatively during revision surgery was positive for aerobic and anaerobic organisms.

When reviewing all of the data from the algorithm protocol, the authors of this study identified a $21.7 \%$ incidence of PJI in the study group.

\section{Discussion}

The diagnosis of PJI can be difficult and requires the application of many different diagnostic strategies 


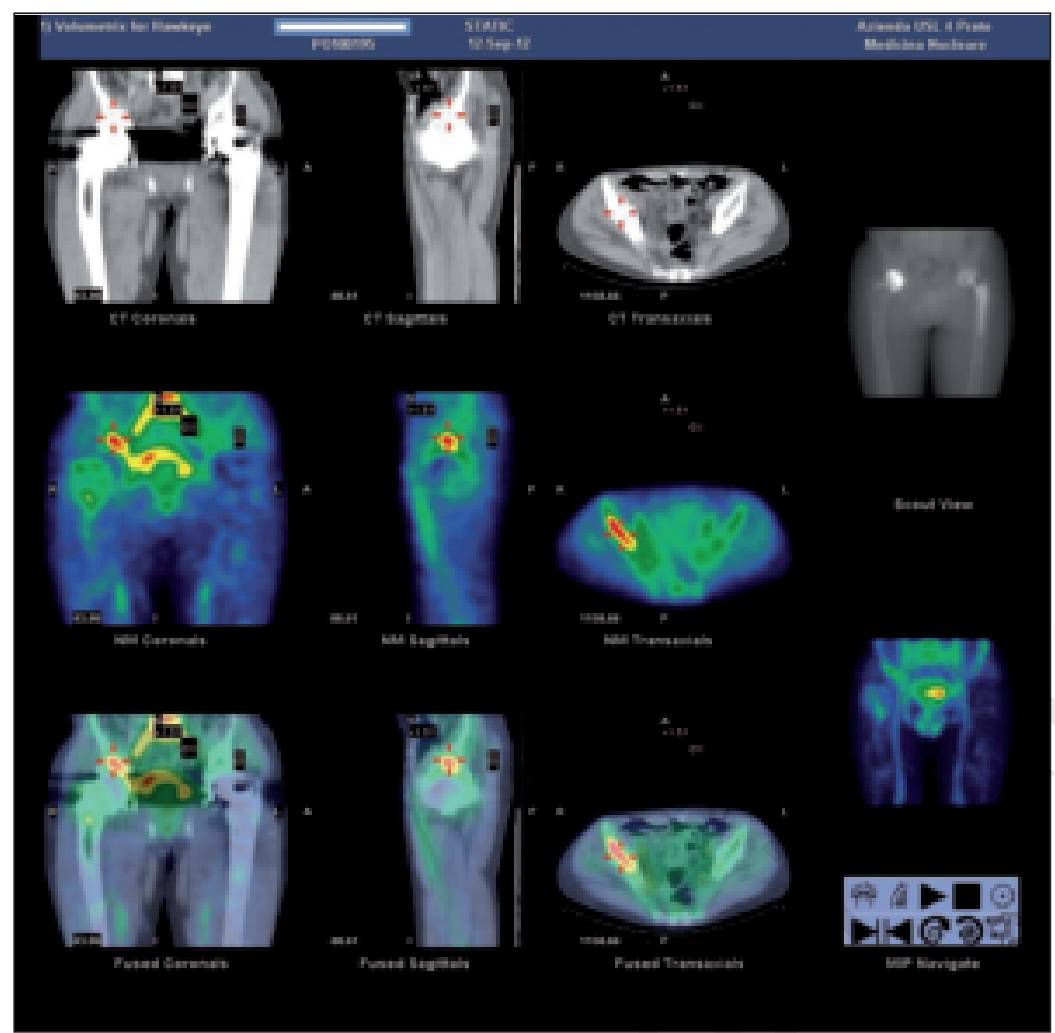

Fig. 2. A ${ }^{99} \mathrm{mTc}-S u l e s u m a b$ bone scan (LeukoScan) with dual time acquisition protocol and SPECT-CT technique with a preliminary finding of increased contrast medium uptake at the level of the trochanteric and periacetabular regions. At 18 hours from contrast medium infusion increased uptake can be noted in the right trochanteric and periacetabular regions. These findings indicated a possible P $\mathrm{JI}$.

including serological, radiographic and microbiological tests. Unfortunately, the management of PJI often necessitates surgical intervention and prolonged courses of intravenous and oral antimicrobial therapy. The authors' standard treatment of a PJI includes implant removal, positioning of an antibiotic-loaded cement spacer, and secondary revision surgery (Fig. 3).

Despite a significant amount of basic and clinical research, an optimal diagnostic pathway for these implant-related infections has yet to be determined. The primary aim of this study was to provide orthopedic surgeons with practical guidelines that address the current controversies in the diagnosis of PJI; these guidelines are aimed specifically at surgeons working in medical institutions that do not have a complete multidisciplinary support group [e.g., infectious disease specialists, general internists, pathologists for intraoperative analysis of frozen sections (20), and fluid biomarker analysts].
The authors of the current study, when approaching a painful TJA, consider exclusion of a PJI as the first step in the differential diagnostic workup. The current literature is controversial because no a single test has been shown to have sufficient sensitivity and/or specificity to confirm a possible PJI (11-16), underlining the need to perform multiple tests (19-24).

Recently, the American Academy of Orthopaedic Surgeons (AAOS) $(9,15)$ released new guidelines in order to allow early diagnosis of PJI. The authors of the current study used some of these recommendations in their own diagnostic algorithm, which was performed in a consecutive case series. This algorithm regards the following preoperative tests as mandatory: 1) serum CRP and ESR levels; 2) standard ${ }^{99} \mathrm{~m}$-technetium MDP bone scan and (only if this test gives a positive result for implant loosening) scanning with ${ }^{99} \mathrm{mTc}$ antigranulocyte antibody Fab’ fragments (LeukoScan); 3) arthrocentesis with analysis of synovial fluid including total cell count and differential leucocyte count and cultures for aerobic and anaerobic organisms. This procedure has shown sufficient sensitivity and specificity in multiple studies (11-14, 16, 19, 21-23).

Intraoperative testing was not considered mandatory by the current authors, but we recognize the validity of intraoperative frozen-section testing, with a positive result in the case of five or more WBC counts per high power field (HPF), positive intraoperative cell count, and differential leucocyte count (20, 24-26).

The diagnosis of PJI has recently reached new milestones. The use of intraoperative or perioperative polymerase chain reaction (PCR) hybridization (27), also in conjunction with implant sonication (28), is showing good correlation with infection severity. There is also substantial evidence on the existence of an innate immune response to pathogens. Microarray techniques have demonstrated an unique gene expression signature exhibited by synovial fluid WBCs from infected joints (29). This unique response to infection 


\section{A}

(D)
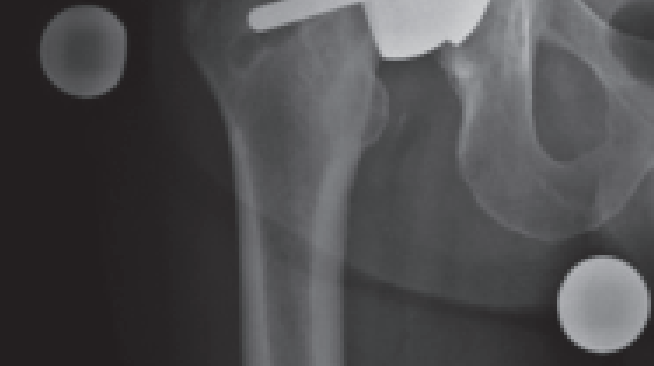

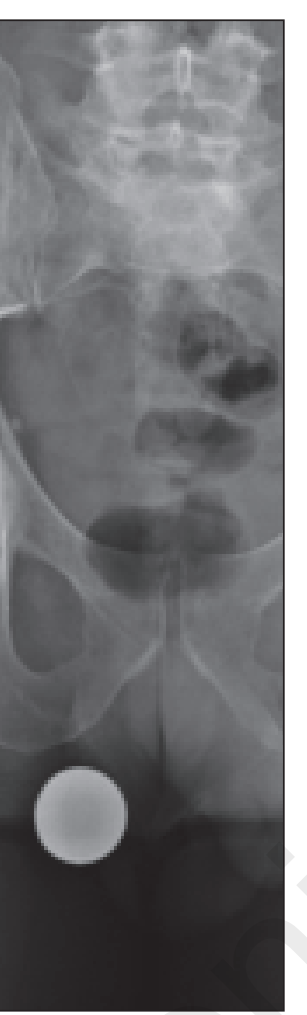

C

(D)

\section{B}

(D)

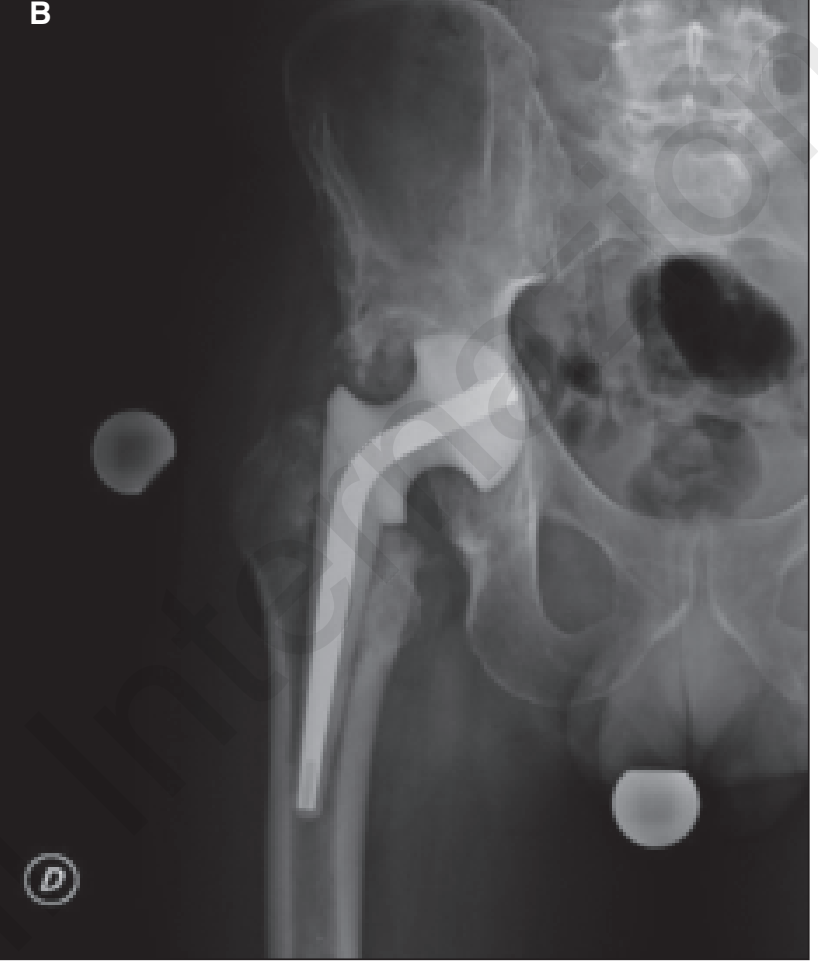

Fig. 3. A forty-nine-year-old male with a painful right total hip arthroplasty at 8 years of follow-up. A: Radiographic evaluation shows loosening of the femoral component and an osteolytic lesion in the periacetabular region. B: Postoperative right hip AP x-ray. An antiperiacetabular region. B: Postoperative right hip AP x-ray. An anti-
biotic spacer is in place. C: The antibiotic spacer has been removed and a revision implant has been positioned. 
was also confirmed at the level of the proteome, with several biomarkers (i.e. IL-6, IL-8, IL-10, IL-17, Lactoferrin, etc.) found to diagnostically outperform the currently available tests for PJI $(30,31)$.

In conclusion, our study showed that the diagnosis of PJI requires the application of a multimodal diagnostic protocol in order to avoid complications related to surgical revision of a misdiagnosed "silent" PJI.

The authors suggest a stepwise diagnostic algorithm, which is designed to be applied in institutions not specifically dedicated to joint surgery: it includes the minimal steps necessary for the diagnosis of PJI.

\section{References}

1. Zimmerli W. Infection and musculoskeletal conditions: prosthetic-joint-associated infections. Best Pract Res Clin Rheumatol. 2006;20:1045-1063.

2. Bozic KJ, Kurtz SM, Al E, Ong K, et al. The epidemiology of revision total knee arthroplasty in the United States. Clin Orthop Relat Res. 2010;468:45-51.

3. Bozic KJ, Kurtz SM, Lau E, Ong K, et al. The epidemiology of revision total hip arthroplasty in the United States. J Bone Joint Surg Am. 2009;91:128-133.

3. Babkin Y, Raveh D, Lifschitz M, et al. Incidence and risk factors for surgical infection after total knee replacement. Scand J Infect Dis. 2007;39:890-895.

4. Carulli C, Matassi F, Civinini R, Villano M, Innocenti M. Surgical prosthetic treatment. Clin Cases Miner Bone Metab. 2010;7:32-38

5. Della Valle CJ, Zuckerman JD, Di Cesare PE. Periprosthetic sepsis. Clin Orthop Relat Res. 2004;(420):26-31.

6. Pulido L, Ghanem E, Joshi A, et al. Periprosthetic joint infection: the incidence, timing, and predisposing factors. Clin Orthop Relat Res. 2008;466:1710-1715.

7. Del Pozo JL, Patel R. Infection associated with prosthetic joints. N Engl J Med. 2009;361:787-794.

8. Matar Y, Jafari M, Restapo C, et al. Preventing infection in total joint arthroplasty. J Bone Joint Surg Am. 2010;92 Suppl 2:36-46.

9. The diagnosis of periprosthetic joint infections of the hip and the knee guideline and evidence report. AAOS - Adopted by the American Academy of Orthopaedic Surgeons. June 18, 2010.

10. Lachiewicz PF, Bolognesi PM, Lonner JH, Parvizi J. Innovative techniques and frontiers in revision TKA Instructional Course 2012.

11. Bedair H, Parvizi J, Jacovides C, et al. The Mark Coventry Award: diagnosis of early postoperative TKA infection using synovial fluid analysis. Clin Orthop Relat Res. 2011;469:3440

12. Della Valle CJ, Sporer SM, Jacobs JJ, Berger RA, Rosenberg AG, Paprosky WG. Preoperative testing for sepsis before revision total knee arthroplasty. J Arthroplasty. 2007;22(6 Suppl 2):90-93.

13. Sofka CM, Potter HG, Adler RS, Pavlov H. Musculoskeletal imaging update: current applications of advanced imaging techniques to evaluate the early and long-term complications of patients with orthopedic implants. HSS J. 2006;2:73-77.
14. Parvizi J, Zmistowski B, Berbari EF, Bauer TW, et al. New definition for periprosthetic joint infection: from the Workgroup of the Musculoskeletal Infection Society. Clin Orthop Relat Res. 2011;469: 2992-2994.

15. Parvizi J, Adeli B, Zmistowski B, Restrepo C, Greenwald AS. Management of periprosthetic joint infection: the current knowledge AAOS exhibit selection. J Bone Joint Surg Am. 2012;94:e104.

16. Osmon DR, Berbari EF, Berendt AR, Lew D, Zimmerli W, Steckelberg JM, Rao N, Hanssen A, Wilson WR. Diagnosis and management of prosthetic joint infection: clinical practice guidelines by the Infectious Diseases Society of America. Clin Infect Dis. 2013; 56:e1-e25.

17. Merchant AC, Mercer RL, Jacobsen RH, Cool CR. Roentgenographic analysis of patellofemoral congruence. J Bone Joint Surg Am. 1974;56:1391-1396.

18. Ryan PJ. Leukoscan for orthopaedic imaging in clinical practice. Nucl Med Commun. 2002;23:707-714.

19. Schinsky MF, Della Valle C, Sporer M, Paprosky WG. Perioperative testing for joint infection in patients undergoing revision total hip arthroplasty. J Bone Joint Surg Am. 2008; 90:1869-1875.

20. Campbell P, Mira J, Doorn P, et al. Histopathology of metal on metal hip joint tissues. In: Rieker C, et al. (Eds) World Tribology Forum in Arthroplasty. 2000;167-180.

21. Berbari E, Marry T, Tsaras G, et al. Inflammatory blood laboratory levels as markers of prosthetic joint infection: a systematic review and meta-analysis. J Bone Joint Surg Am. 2010;92:2102-2109.

22. Bilgen O, Atici T, Durak K, Karaeminoğullari, Bilgen MS. C-reactive protein values and erythrocyte sedimentation rates after total hip and total knee arthroplasty. J Int Med Res. 2001;29:7-12.

23. Larsson S, Thelander U, Friberg S. C-reactive protein (CRP) levels after elective orthopedic surgery. Clin Orthop Relat Res. 1992;(275):237-242.

24. Tohtz SW, Müller M, Morawitz L. Validity of frozen sections for analysis of periprosthetic loosening membranes. Clin Orthop Relat Res. 2010;468:762-768.

25. Trampuz A, Hanssen AD, Osmon DR, Mandrekar J, Steckelberg JM, Patel R. Synovial fluid leukocyte count and differential for the diagnosis of prosthetic knee infection. Am J Med. 2004;117:556-562.

26. Müller M, Morawietz L, Hasart O, et al. Diagnosis of periprosthetic infection following total hip arthroplasty-evaluation of the diagnostic values of pre- and intraoperative parameters and the associated strategy to preoperatively select patients with a high probability of joint infection. J Orthop Surg Res. 2008;3:31.

27. Mariani BD, Martin DS, Levine MJ, Booth RE Jr, Tuan RS. The Coventry Award. Polymerase chain reaction detection of bacterial infection in total knee arthroplasty. Clin Orthop Relat Res. 1996;(331):11-22.

28. Esteben J, Alonso-Rodriguez N, del-Prado G, et al. PCRhybridization after sonication improves diagnosis of implantrelated infection. Acta Orthop. 2012;83:299-304.

29. Parvizi J, Jacovides C, Zmistowski B, Jung KA. Definition of periprosthetic joint infection: is there a consensus? Clin Orthop Relat Res. 2011;469:3022-3030.

30. Deirmengian C, Hallab N, Tarabishy A, et al. Synovial fluid biomarkers for periprosthetic infection. Clin Orthop Relat Res. 2010;468:2017-2023.

31. Jacovides CL, Parvizi J, Adeli B, et al. Molecular markers for diagnosis of periprosthetic joint infection. J Arthroplasty. 2011;26(6 Suppl):99-103. 